\title{
A preliminary study on the relationship between energy expenditure and skin temperature in swimming
}

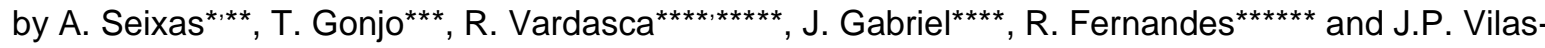 \\ Boas $^{\star \star * \star * \star *}$ \\ * Universidade Fernando Pessoa, Porto, Portugal, aderito@ufp.edu.pt \\ ** LABIOMEP, CIAFEL, Faculty of Sport, University of Porto, Portugal \\ *** Centre for Aquatics Research and Education, The University of Edinburgh, Edinburgh, Scotland, UK \\ **** IDMEC-FEUP, LABIOMEP, Faculty of Engineering, University of Porto, Portugal \\ $\star \star \star \star \star$ Medical Imaging Research Unit, Faculty of Computing, Engineering and Science, University of South \\ Wales, Wales, UK

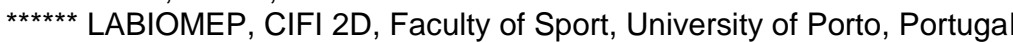

\begin{abstract}
This study experimentally investigated the possible relationship between energy expenditure and skin temperature during $2 \times 300 \mathrm{~m}$ protocols (front crawl and backstroke), performed at the same intensity and velocity, in two national level male swimmers. Both swimming techniques increased skin temperature but with different thermal patterns. Higher values of energy expenditure during the $300 \mathrm{~m}$ backstroke test were found comparing with the values obtained for the same distance performed in front crawl and higher difference between skin temperatures values were associated with lower energy expenditure values.
\end{abstract}

\section{Introduction}

Thermography is a non-invasive, non-ionizing and non-radiation emitting imaging modality, which records the surface temperature of an object. Skin temperature (Tsk) is influenced by heat exchange processes between skin, deep tissues, local blood vessels and metabolism [1] and the validity and reliability of this method to measure Tsk has been previously documented [2]. Its applicability in sports science is not widespread despite early records [3].

As any other sport, swimming induces changes to the thermal balance of the human body. Part of the heat produced during the exercise is given off by the skin through a complex thermoregulation process, resulting in an increase of skin temperature [4] and maintaining the central temperature close to $37.5^{\circ} \mathrm{C}$ [5]. Infrared thermography can be used to analyse the thermoregulation process, giving insight on the dissipation process of heat produced by muscular activity [6]. A low number of investigations have attempted to assess the skin temperature of swimmers or the effects of different swimming techniques in the Tsk distribution of swimmers. For instance, Zaïdi et al. [4] studied the influence of the swimming technique on the skin temperature maps of a single competitive swimmer using infrared thermography. This technology is emerging in the field of sport sciences but only that study and another [7] mentioned the application of thermography to swimming activities.

Energy expenditure is a widely used parameter in swimming science as an indicator of performance [810].Thermal imaging has been used in cycling to explore the relationship between skin temperature and gross efficiency [5] but, to our knowledge, no study has used infrared thermography to investigate the possible relationship between energy expenditure and skin temperature in swimming activities. The aims of this research are to study the influence of two different swimming techniques in the Tsk distribution and analyse the relationships between Tsk and energy expenditure in national level competitive swimmers.

\section{Methodology}

\subsection{Subjects}

Two national level, well-trained male swimmers (18 years old; $21.8 \pm 2.2 \mathrm{Kg} / \mathrm{m}^{2}$ ) volunteered to serve as subjects. Both swimmers reported training 1.5-3h each session, 6-9 times per week. The study protocol was previously approved by the local ethics committee and the rules of the declaration of Helsinki were followed. Both swimmers were informed about the experimental procedure, the potential risks and benefits and gave written consent to participate in the study.

\subsection{Experimental procedures}

A cross-sectional study was planned in order to collect energy expenditure data during $2 \times 300 \mathrm{~m}$ protocols (the $1^{\text {st }}$ bout at backstroke and the $2^{\text {nd }}$ at front crawl) and thermal data. Each participant reported for testing for two days, at 
the same time of the day, one for each experimental condition. All tests were conducted in a 25-m indoor pool with water temperature of $26.5 \pm 0.5^{\circ} \mathrm{C}$, air temperature of $26.1 \pm 0.4^{\circ} \mathrm{C}$, relative humidity of $74.4 \pm 3.1 \%$ and no airflow.

During each visit, two Tsk measurements were performed, one before the experimental condition and one after the experimental condition. Full body thermograms were obtained with a thermographic camera (FLIR A325, FLIR Systems, Wilsonville, USA), properly calibrated, presenting resolution of $320 \times 240$, sensitivity of $70 \mathrm{mK}$ and accuracy of $\pm 2 \%$. Thermograms capture and analysis were made using the software FLIR ThermaCAM Researcher Pro $2.10^{\circledR}$. For data analysis the skin surface was divided into polygonal surfaces, defining 18 regions of interest (figure 1). The right and left arms, forearms, thighs, legs and trunk (in anterior and posterior views) were defined as regions of interest. Average Tsk was calculated over the zones as a whole, as the sum of the product of the mean temperature of each region of interest and the number of pixels of each zone, all divided by the sum of the number of pixels of every region of interest [4].

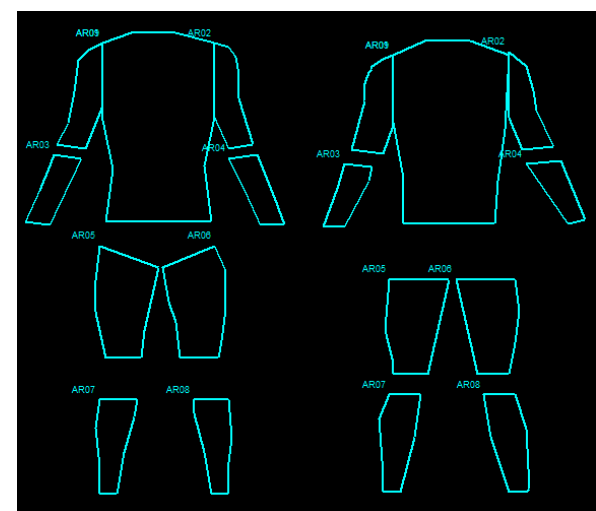

Figure 1. Regions of interest selected in anterior view (left) and posterior view (right)

In both experimental sessions each swimmer was asked to stay in a static position for 10 minutes, immersed in the water up to the neck. After this acclimatization period, the swimmer left the pool and was rapidly dried with a microfiber towel, without friction to avoid heating. The first set of thermograms was recorded with full anterior and posterior body images. The first set of images constitutes the thermal reference level at rest. The swimmer then performed the 300m test, backstroke or front crawl and at the end was rapidly dried and the second set of thermograms was obtained. The testing was conducted at the same intensity and velocity for both front crawl and backstroke, controlled by a visual light pacer (pacer 2 swim, KulzerTEC, Santa Maria da Feira, Portugal). During the 300m bouts the swimmers breathed through a respiratory snorkel AquaTrainer and valve system connected to a telemetric portable gas analyzer (K4b2, Cosmed, Rome, Italy) for oxygen uptake $\left(\mathrm{VO}_{2}\right)$ recording and energy expenditure calculation $\left(\mathrm{mlO}_{2} / \mathrm{kg} / \mathrm{m}\right)$.

\section{Results}

Figures 2, 5 and 6 illustrate both swimmers after the acclimatization period, after the front crawl protocol and after the backstroke protocol, respectively.

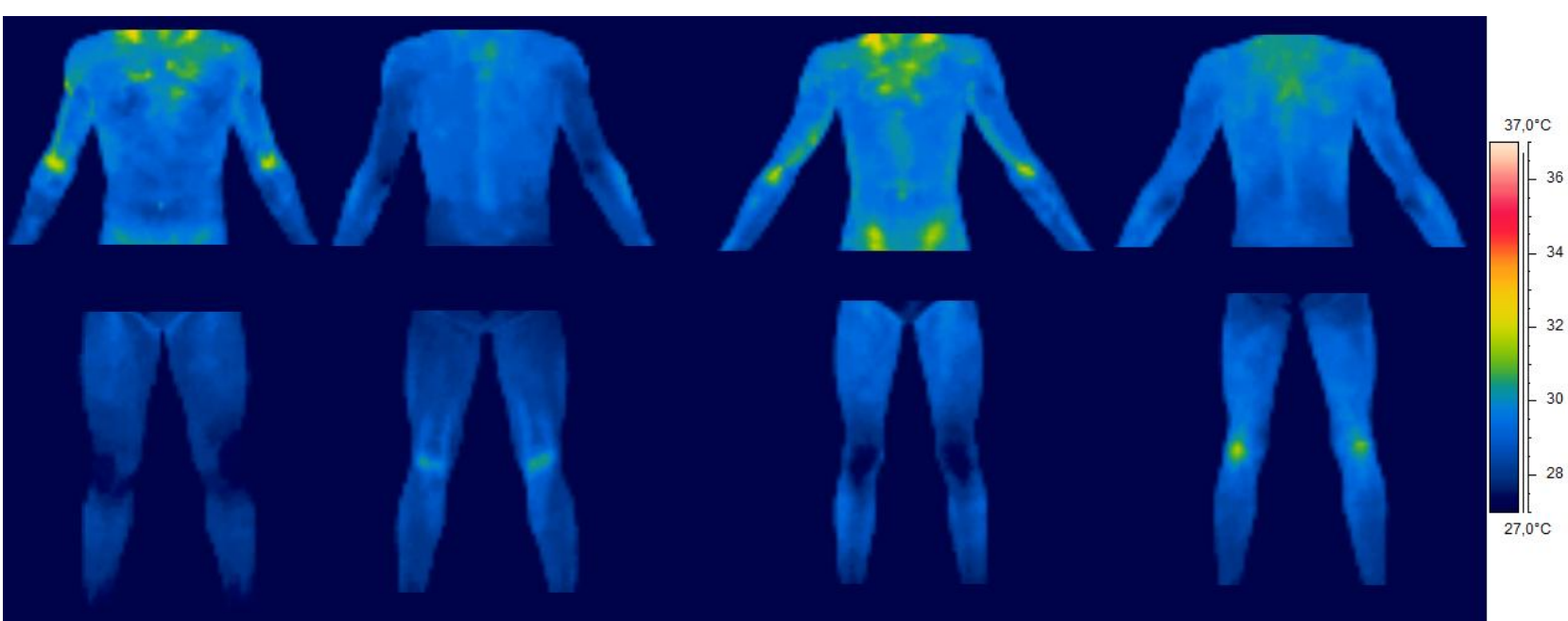

Figure 2. Frontal and dorsal views: baseline thermograms of swimmer 1 (1 $1^{\text {st }}$ and $2^{\text {nd }}$ images images) and swimmer 2 (3 $3^{\text {ra }}$ and $4^{\text {th }}$ images) 
Reference Tsk values of each region of interest are shown in figure 3 being observed higher Tsk values on the trunk and arms and lower values in the legs of both swimmers. Thermal patterns of both swimmers changed after exercise. The backstroke swimming technique was responsible for more generalized changes, as shown in figure 6 . Total Tsk after the backstroke protocol was higher in both swimmers.

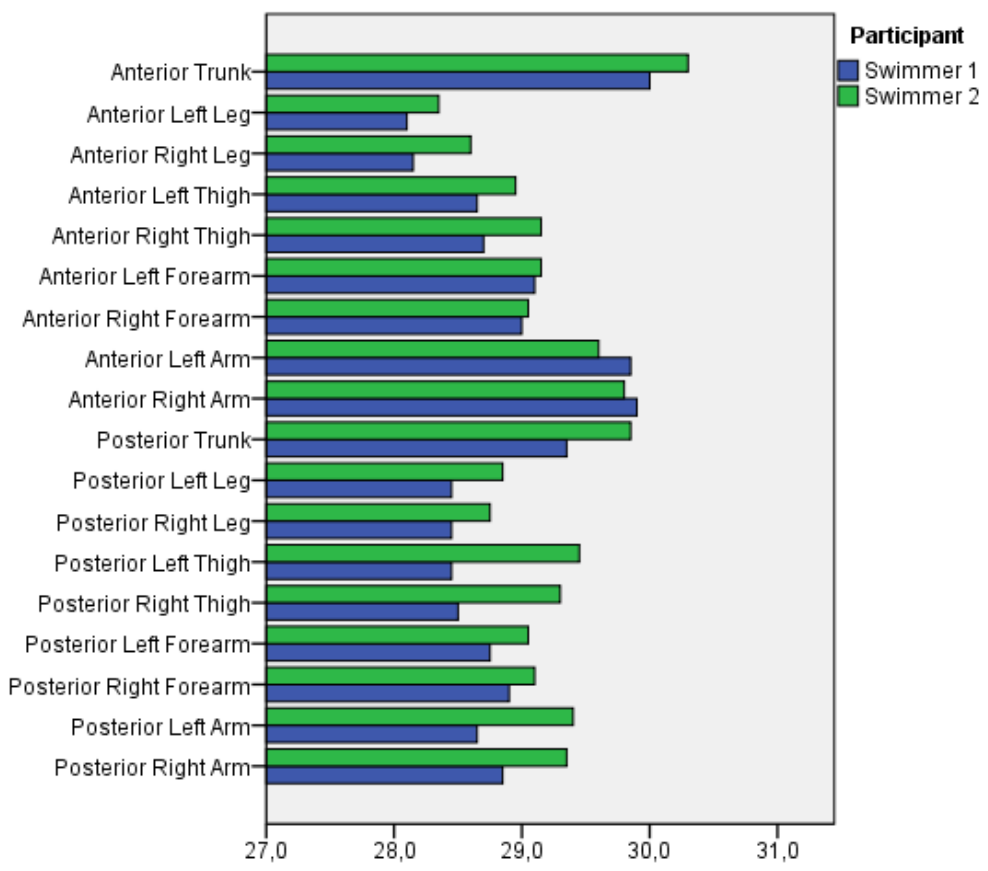

Figure 3. Reference skin temperature values $\left({ }^{\circ} \mathrm{C}\right)$ of each region of interest for both swimmers

Thermal variation in each region of interest is shown in figure 4 and evidenced different tendencies in both swimmers, with swimmer 1 evidencing higher thermal variation after the front crawl bout and swimmer 2 presenting higher thermal variation after the backstroke swim.
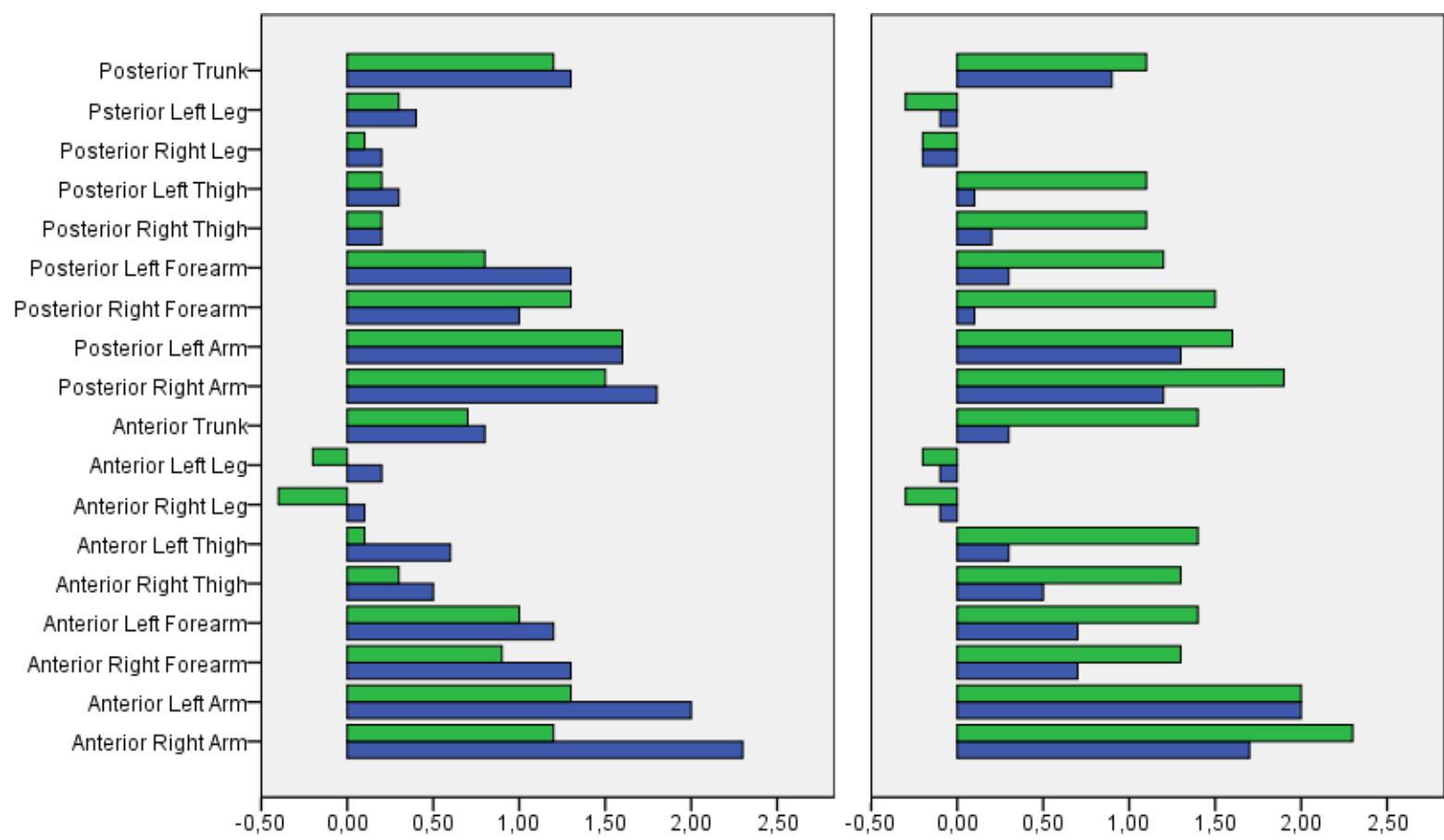

Figure 4. Regional thermal variation $\left({ }^{\circ} \mathrm{C}\right)$ in swimmer 1 (left) and swimmer 2 (right) 
The lower limbs were less affected by exercise than the upper limbs and trunk in both exercise protocols. In the anterior surface of the legs, in both swimmers, Tsk values decreased when compared to baseline and in the posterior aspect of the legs Tsk values decreased in swimmer 2 after both protocols.

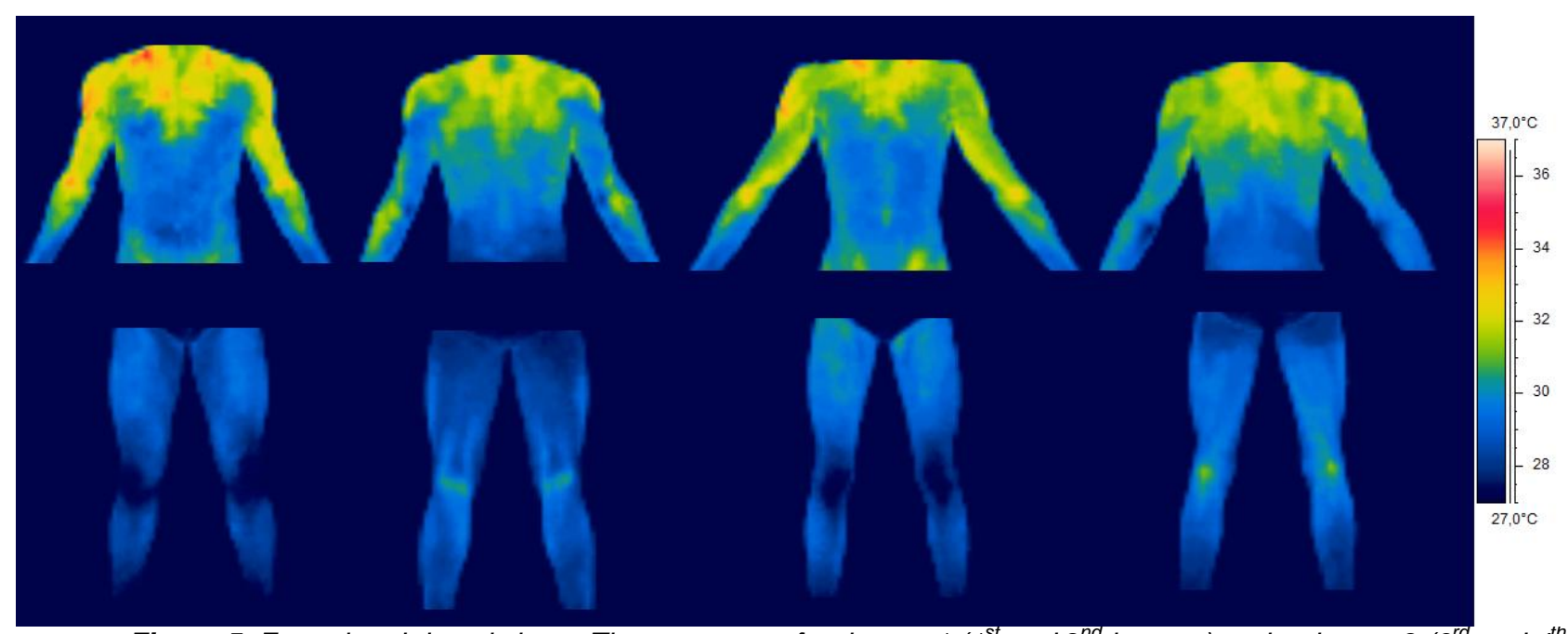

Figure 5. Frontal and dorsal views: Thermograms of swimmer $1\left(1^{\text {st }}\right.$ and $2^{\text {nd }}$ images) and swimmer $2\left(3^{\text {rd }}\right.$ and $4^{\text {th }}$ images) after the front crawl bout

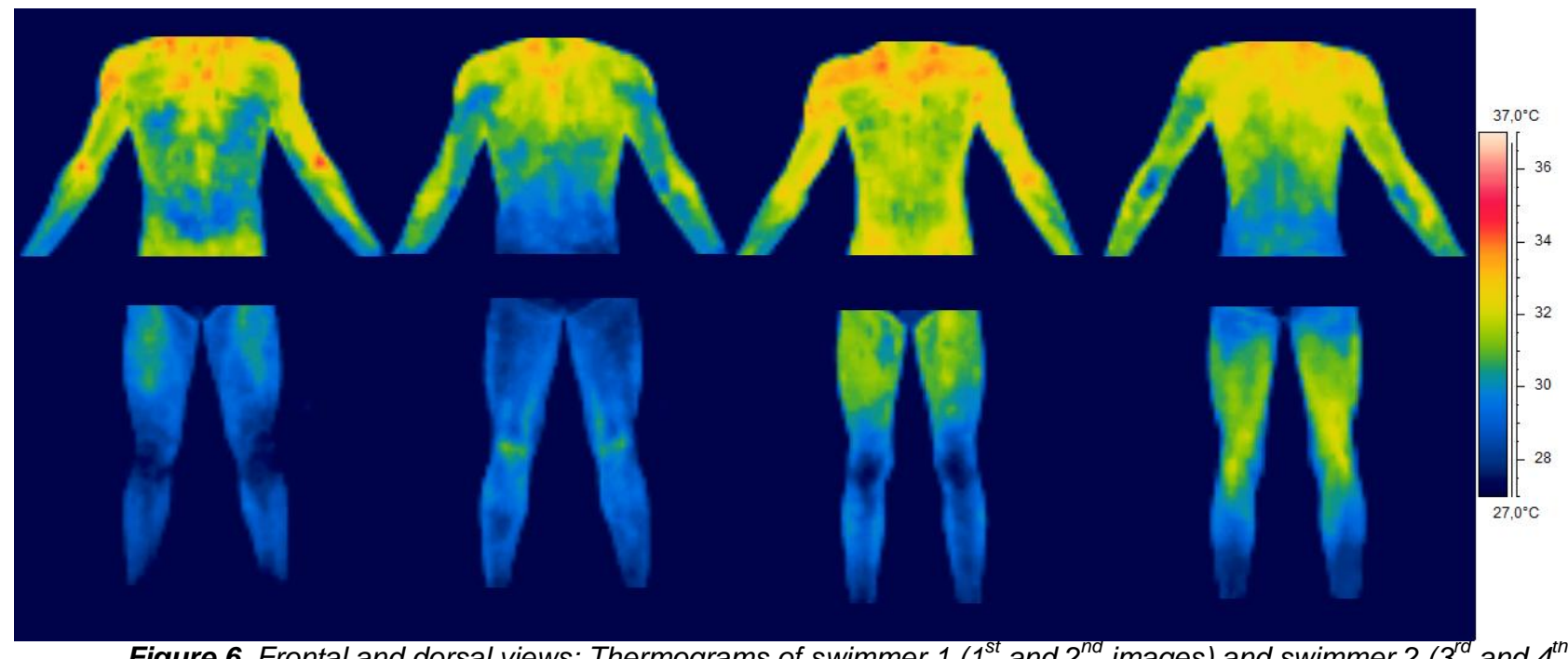

Figure 6. Frontal and dorsal views: Thermograms of swimmer $1\left(1^{\text {st }}\right.$ and $2^{\text {nd }}$ images) and swimmer $2\left(3^{\text {rd }}\right.$ and $4^{\text {th }}$ images) after the backstroke bout

In table 1, the values of total Tsk before and after testing, as well as the energy expenditure values are presented for both swimmers for front crawl and backstroke, respectively.

Table 1. Total skin temperature variation and energy expenditure in front crawl and backstroke protocols at the same

\begin{tabular}{lccccc}
\hline & velocity \\
\hline Velocity $(\mathrm{m} / \mathrm{s})$ & $\begin{array}{c}\text { Total Tsk } \\
\text { before protocol } \\
\left({ }^{\circ} \mathrm{C}\right)\end{array}$ & $\begin{array}{c}\text { Total Tsk after } \\
\text { protocol }\left({ }^{\circ} \mathrm{C}\right)\end{array}$ & $\Delta$ Tsk $\left({ }^{\circ} \mathrm{C}\right)$ & $\begin{array}{c}\text { Energy } \\
\text { Expenditure } \\
\left(\mathrm{mlO}^{2} / \mathrm{kg} / \mathrm{min}^{2}\right)\end{array}$ \\
\hline Swimmer 1 (front crawl) & 1.06 & 28.76 & 29.66 & 0.90 & 39.15 \\
Swimmer 2 (front crawl) & 1.11 & 29.23 & 29.79 & 0.56 & 40.10 \\
Swimmer 2 (backstroke) & 1.06 & 29.46 & 30.16 & 0.70 & 55.66 \\
\hline
\end{tabular}


Total Tsk increased after both swim exercises in both swimmers and energy expenditure was higher for backstroke in both swimmers. Looking at each swimming technique, lower values of total Tsk variation corresponded to higher values of energy expenditure.

\section{Discussion}

To date, only two studies tried to evidence the applicability of infrared thermography in swimming and the influence of the swimming technique in cutaneous temperature. One of those studies [7] used a thermal imaging system with low sensitivity and with insufficient software and tools and the other assessed the effects of different swimming techniques on the distribution of skin temperature in one swimmer [4].

The lack of research in this field is certainly related to the environmental conditions in which swimmers perform. The temperature of the swimming pool exceeded the recommendations from previous literature [11, 12] and relative humidity was high, but the athletes practice their sport in these conditions and should be studied likely. Data was collected before and after exercise under the same environmental conditions, reducing the possible bias.

At baseline, in both swimmers, higher temperatures were found in the trunk (anterior and posterior views) and anterior aspect of the right and left arms, and lower temperatures were reported in the anterior aspects of the legs. Zaidi et al. [4] reported higher reference values in the trunk, anterior aspect of the arms, thigh and posterior aspect of the legs and lower values in the forearms. It might be argued that the differences may be related with individual variations in skin temperature, but in our study both swimmers revealed the same tendency. The skin drying protocol was not detailed in the above mentioned study and might have been responsible for these differences. In our study, after the acclimatization period in the water the swimmers were rapidly dried with a microfiber towel without friction to avoid heating.

Tsk increased after performing each of the swimming techniques but the thermal pattern was different in between. Higher variation was observed in the trunk and upper limbs and lower variations in Tsk were observed in the lower limbs. Considering the data collected from both swimmers, backstroke bout was responsible for higher increments in total Tsk in agreement with previous published literature [4]. Zaidi et al. have addressed the influence of butterfly, backstroke, breaststroke and freestyle on the distribution of Tsk.

Previous studies have demonstrated that front crawl and backstroke techniques were more economical than butterfly and breast stroke and that front crawl was more economical than backstroke at slower and maximal speeds [9, $13,14]$. Our results are in line with these studies and confirmed higher energy expenditure values during backstroke test than during front crawl test.

This was the first attempt to study the relationship between energy expenditure and Tsk variation in different swimming techniques. Considering each swimming technique independently, higher values of energy expenditure corresponded to lower values of Tsk variation. Bertucci et al. [5] published a preliminary study on the relationship between gross efficiency, given as the quotient of power output and metabolic power, and skin temperature of the lower limbs in cycling. The authors recruited 2 cyclists and used an incremental test, starting at $100 \mathrm{~W}$ and increased $40 \mathrm{~W}$ every 4 minutes until exhaustion. At the same power output, the cyclist with lower gross efficiency, and thus higher energy expenditure, produced less heat than the cyclist with higher gross efficiency. No similar study was found in swimming activities but our results are in line with the above referred study, when looking at each technique independently.

\section{Conclusion}

In both swimmers, the studied swimming techniques increased Tsk, with distinct thermal patterns. Higher differences between total Tsk before exercise and after exercise seem to be associated with lower values of energy expenditure. These findings highlight the potential use of Tsk as a physiological indicator of energy expenditure but should be confirmed with a study with a high number of swimmers. The role of age, gender and body mass index in the relationship between Tsk and energy expenditure should be explored, providing important reference data for future studies regarding performance and sport medicine.

\section{AKNOWLEDGEMNT}

This research is sponsored by FEDER funds through the program COMPETE - Programa Operacional Factores de Competitividade and by national funds through FCT-Fundação para a Ciência e a Tecnologia, under the Project "AAL4ALL - Padrão de Cuidados Primários para Serviços de AAL" (FCOMP-01-0124-FEDER-13852) co-financed by FEDER, Programa Operacional Factores de Competitividade.

\section{REFERENCES}

[1] Merla, A. and Romani, G., "Biomedical applications of functional infrared imaging" presented at the 27th Annual International Conference of the Engineering in Medicine and Biology Society, Shanghai (China), 2005.

[2] Burnham, R. S., McKinley, R. S., and Vincent, D. D., "Three types of skin-surface thermometers: a comparison of reliability, validity, and responsiveness". American journal of physical medicine \& rehabilitation, vol. 85, pp. 553-558, 2006. 
[3] Hunold, S., Mietzsch, E., and Werner, J., "Thermographic studies on patterns of skin temperature after exercise". European journal of applied physiology and occupational physiology, vol. 65, pp. 550-554, 1992.

[4] Zaidi, H., Fohanno, S., Polidori, G., and Taiar, R., "The influence of swimming type on the skin-temperature maps of a competitive swimmer from infrared thermography". Acta of Bioengineering and Biomechanics, vol. 9, p. 47, 2007.

[5] Bertucci, W., Arfaoui, A., Janson, L., and Polidori, G., "Relationship between the gross efficiency and muscular skin temperature of lower limb in cycling: a preliminary study". Computer methods in biomechanics and biomedical engineering, vol. 16, pp. 114-115, 2013.

[6] Arfaoui, A., Polidori, G., Taïar, R., and Popa, C., "Infrared thermography in sports activity," in Infrared Thermography, R. Prakah, Ed., ed: Intech, 2012, pp. 141-168.

[7] Wade, C. and Veghte, J., "Thermographic evaluation of the relative heat loss by area in man after swimming". Aviation, space, and environmental medicine, vol. 48, pp. 16-18, 1977.

[8] Smith, D. J., Norris, S. R., and Hogg, J. M., "Performance evaluation of swimmers". Sports Medicine, vol. 32, pp. 539-554, 2002.

[9] Barbosa, T., Fernandes, R., Keskinen, K., Colaço, P., Cardoso, C., Silva, J., et al., "Evaluation of the Energy Expenditure in Competitive Swimming Strokes". International Journal of Sports Medicine, vol. 27, pp. 894-899.

[10] Fernandes, R., Billat, V., Cruz, A., Colaço, P., Cardoso, C., and Vilas-Boas, J., "Does net energy cost of swimming affect time to exhaustion at the individual's maximal oxygen consumption velocity?". The Journal of sports medicine and physical fitness, vol. 46, p. 373, 2006.

[11] Schwartz, R., "Guidelines for neuromusculoskeletal thermography". Thermology international, vol. 16, pp. 5-9, 2006.

[12] Ring, E. and Ammer, K., "The technique of infrared imaging in medicine". Thermology international, vol. 10, pp. 7-14, 2000

[13] Holmér, l., "Energy cost of arm stroke, leg kick, and the whole stroke in competitive swimming styles". European journal of applied physiology and occupational physiology, vol. 33, pp. 105-118, 1974.

[14] Capelli, C., Pendergast, D. R., and Termin, B., "Energetics of swimming at maximal speeds in humans". European journal of applied physiology and occupational physiology, vol. 78, pp. 385-393, 1998. 